Original research article

\title{
Atorvastatin prevents the development of diabetic neuropathic nociception by possible involvement of nitrergic system
}

\author{
Reyhaneh Akbarian ${ }^{1,2}$, Mohsen Chamanara ${ }^{3}$, Amir Rashidian ${ }^{1,2}$, Alireza Abdollahi ${ }^{4}$, \\ Shahram Ejtemaei Mehr ${ }^{1,2}$, Ahmad Reza Dehpour ${ }^{1,2}$ * \\ ${ }^{1}$ Tehran University of Medical Sciences, Experimental Medicine Research Center, Tehran, Iran \\ ${ }^{2}$ Tehran University of Medical Sciences, School of Medicine, Department of Pharmacology, Tehran, Iran \\ ${ }^{3}$ Aja University of Medical Sciences, Faculty of Medicine, Department of Pharmacology, Tehran, Iran \\ ${ }^{4}$ Tehran University of Medical Sciences, Imam Hospital complex, Department of Pathology, Tehran, Iran
}

\begin{abstract}
Aims: Diabetic neuropathy has been identified as a common complication caused by diabetes. However, its pathophysiological mechanisms are not fully understood yet. Statins, also known as HMG-CoA reductase inhibitors, alleviate the production of cholesterol. Despite this cholesterol-reducing effect of statins, several reports have demonstrated their beneficial properties in neuropathic pain. In this study, we used streptozotocin (STZ)-induced diabetic model to investigate the possible role of nitric oxide (NO) in the antineuropathic-like effect of atorvastatin.

Methods: Diabetes was induced by a single injection of STZ. Male rats orally received different doses of atorvastatin for 21 days. To access the neuropathy process, the thermal threshold of rats was assessed using hot plate and tail-flick tests. Moreover, sciatic motor nerve conduction velocity (MNCV) studies were performed. To assess the role of nitric oxide, N(G)-nitro-L-arginine methyl ester (L-NAME), aminoguanidine (AG), and 7-nitroindazole (7NI) were intraperitoneally administered along with some specific doses of atorvastatin.

Key findings: Atorvastatin significantly reduced the hyperalgesia in diabetic rats. L-NAME pretreatment with atorvastatin showed the antihyperalgesic effect, suggesting the possible involvement of the NO pathway in atorvastatin protective action. Furthermore, coadministration of atorvastatin with AG and 7NI resulted in a significant increase in pain threshold in diabetic rats.

Significance: Our results reveal that the atorvastatin protective effect on diabetic neuropathy is mediated at least in a part via the nitric oxide system.
\end{abstract}

Keywords: Atorvastatin; Diabetic neuropathy; Hyperalgesia; Nitric oxide; Rat

\section{Highlights:}

- Atorvastatin administration attenuates diabetic neuropathy.

- Atorvastatin exerts anti-neuropathic properties through the nitrergic system.

- iNOS is involved in diabetic neuropathy.

\section{Introduction}

Peripheral neuropathy is a frequent, devastating and costly complication caused by diabetes, which has been recognized as one of the most challenging types of pain to treat (Kaur et al., 2011; Rosenberger et al., 2020). Many diabetic patients are suffering from chronic pain, mostly characterized by allodynia (pain perception due to non-painful stimuli) and hyperalgesia (exaggerated responses to painful stimuli), which consequently degrades their quality of life severely (Jesus et al., 2019; Sun et al., 2012). Most often, diabetic neuropathy associated with abnormal nociception would be a progressive disorder. Accord- ing to previous studies, many neural dysfunctions can result from this disorder, including demyelination, axonal degeneration, reduction in density of small unmyelinated fibers, and Wallerian degeneration follows by diabetes (Bhalla et al., 2015; Chu et al., 2015; Heltianu and Guja, 2011). Diabetes induced by streptozotocin (STZ), as a pancreatic beta-cell toxin, is one of the appropriate models to assess the mechanism of diabetes. The most commonly used animal models can be applied to investigate the underlying mechanisms and to evaluate potential therapies of diabetic neuropathy (Eitah et al., 2019; Jesus et al., 2019; Mbiantcha et al., 2020).

Nitric oxide (NO), as a unique neuromodulator molecule, is a diffusible second-messenger with diverse pharmacological

\footnotetext{
* Corresponding author: Ahmad Reza Dehpour, Tehran University of Medical Sciences, School of Medicine, Department of Pharmacology, Tehran, Iran; e-mail: dehpoura@sina.tums.ac.ir; dehpour@yahoo.com http://doi.org/10.32725/jab.2021.006

Submitted: 2020-05-09 • Accepted: 2021-01-28 • Prepublished online: 2021-02-09

J Appl Biomed 19/1: 48-56 • EISSN 1214-0287 • ISSN 1214-021X

(c) 2021 The Authors. Published by University of South Bohemia in České Budějovice, Faculty of Health and Social Sciences.

This is an open access article under the CC BY-NC-ND license.
} 
applications throughout the human body (Hassanipour et al., 2016; Shafaroodi et al., 2012). Basically, NO is synthesized by one of the following three forms of nitric oxide synthases (NOS): neuronal NOS (nNOS), endothelial NOS (eNOS), and inducible NOS (iNOS) (Saha and Pahan, 2006). It has been demonstrated that iNOS activity promotes the pathogeneses of neuroinflammatory and neurodegenerative human diseases. Due to overactivity of iNOS under diabetic conditions, peroxynitrite has been formed, which caused deterioration of regional circulation and the axonal dysfunctions through oxidative modifications on the neural tissue (Ahlawat and Sharma, 2018; Heltianu and Guja, 2011). There is an abundance of evidence indicating that NO may mediate nociceptive information transmission at three levels of peripheral, spinal, and supraspinal (Saffarpour and Nasirinezhad, 2019; Staunton et al., 2018). NO can play a dual role under both pro-inflammatory and anti-inflammatory conditions after nerve damage in different animal models (Cury et al., 2011; de Los Monteros-Zuñiga et al., 2016; Gomes et al., 2020). Therefore, it contributes not only to pain hypersensitivity, but also to the development of analgesia in nociception (Aydin et al., 2013). Previous studies indicated that NO plays pivotal roles in the development and maintenance of hyperalgesia. So, iNOS inhibitors may be implicated in diabetic neuropathic pain by reducing oxidative stress and inflammatory mediators as well as improving demyelination. Several studies have suggested beneficial effects of the selective and non-selective NOS inhibitors on reducing thermal hyperalgesia in inflammatory pain models (Chen et al., 2010; Rondón et al., 2018).

The statins, HMG-CoA (3-hydroxy-3-methyl-glutaryl-coenzyme A) reductase inhibitors, are widely prescribed for the prevention of coronary heart disease (Pathak et al., 2013). These drugs have broad-spectrum and cholesterol independent effects, including anti-inflammatory, anti-oxidant, anti-microbial, immunomodulatory, and neuroprotective properties (Aydin et al., 2013; Bhalla et al., 2015). It is believed that statins could attenuate peripheral and central pains (Pathak et al., 2014). Treatment with statins demonstrates antinociceptive activity in partial sciatic nerve ligation and in rat's trigeminal neuralgia model. Garcia et al. in 2011 found that systemic administration of atorvastatin could cause significant anti-nociception in the tail-flick, writhing, formalin hind paw, and orofacial formalin tests. However, more studies are needed to explore the mechanisms involved in these favorable outcomes (Garcia et al., 2011). Moreover, atorvastatin is believed to prevent cardiovascular disease in patients with type 2 diabetes (Newman et al., 2008; Raikou et al., 2007). In this regard, a study performed on STZ-induced diabetic mice demonstrated that atorvastatin that exhibits an antidepressant effect could inhibit neuroinflammation (Hai-Na et al., 2020). Despite the evidence pointing to the efficacy of atorvastatin on diabetes, there are no studies investigating the role of NO in the anti-neuropathic effect of atorvastatin, so far. In this paper, we aimed to investigate the possible contribution of NO in atorvastatin anti-neuropathic effects on STZ-induced diabetic rats.

\section{Materials and methods}

\section{Animals}

Eighty-four male Wistar rats, weighing 220-260 g were housed under the controlled-temperature $\left(22 \pm 1{ }^{\circ} \mathrm{C}\right)$ condition with regular light/dark cycle. The rats had free access to food and water. All the experiments were performed in terms of the National Institute of Health (NIH) Guidelines for the Care and Use of Laboratory Animals (HHS publication 85-23, 1985), and legislation for the protection of animals used for scientific purposes (Directive 2010/63/EU) in Tehran University of Medical Sciences.

\section{Drugs}

The following drugs were used throughout this study: STZ (Sigma-Aldrich, USA), atorvastatin, an HMG-CoA inhibitor (Sobhan, Iran); N( $\omega)$-nitro-L-arginine methyl ester (L-NAME), a non-specific inhibitor of NOS (Sigma, USA); aminoguanidine (AG), a selective inhibitor of iNOS (Sigma, USA); and 7-nitroindazole (7NI), a selective inhibitor of nNOS (Sigma, USA). Except atorvastatin and 7-NI, all drugs were dissolved in normal saline. Atorvastatin suspension was prepared in carboxymethyl cellulose (CMC, $0.5 \%)$ and then administered orally. $7-\mathrm{NI}$ was suspended in a 1\% aqueous solution of Tween 80 .

\section{Experimental design}

STZ (65 mg/kg i.p.) served as a diabetes-inducer in our study. 24 hours before the administration of STZ, the animals were deprived of food. To assess their blood glucose level, 48 hours after the STZ administration, blood samples were drawn out from the tail and then measured by blood glucose monitoring system (ACCU-CHEK Active, Roche). Accordingly, the animals with blood glucose levels higher than $400 \mathrm{mg} / \mathrm{dl}$ were considered as diabetic (Hasanvand et al., 2016). The animals were randomly assigned into twelve groups; each one consisted of 7 rats. Thereafter, different doses of atorvastatin $(10,20$, and $40 \mathrm{mg} / \mathrm{kg}$ ) (Rashidian et al., 2016) were orally administered per day for a 21-day period (He et al., 2019) (by passing 48 hours from the STZ administration). In order to investigate the involvement of the NO pathway in the diabetic neuropathy, 7-NI (15 mg/kg), L-NAME (5 mg/kg), and AG (50 mg/kg) (Rostamian et al., 2019) were injected i.p. 30 minutes before atorvastatin administration into the rats. The Vehicle group was diabetic animals that received CMC 5\% for 21 days. The control animals were not diabetic, so they were injected with an equal volume of the saline. All behavioral assessments were recorded manually and blinded (Fig. 1).

\section{Survival study and general toxicity}

The general condition of animals, including weight loss and mortality rate, were daily followed up and the rats were weighed weekly throughout the experiment.

Atorvastatin and NOS inhibitors administration

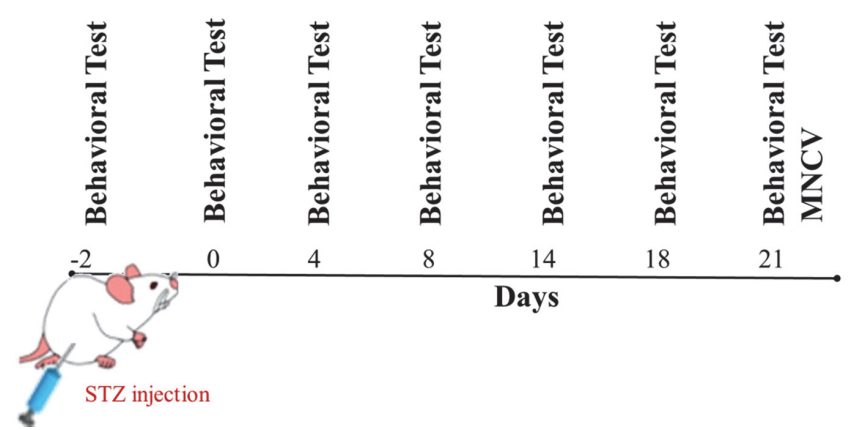

Fig. 1. Outline of the experimental design. See "Materials and methods" section for complete description 


\section{Behavioral tests}

Hot plate test

The thermal nociceptive threshold, as an index of thermal hyperalgesia, was assessed using an electrically-heated surface $\left(55 \pm 1^{\circ} \mathrm{C}\right.$ ) (Tahghigh-Gostaran-Teb, Iran). The rats were separately placed on the hot plate, and then withdrawal latency was recorded in seconds concerning licking of the hind paw. The thermal threshold was measured at the days $-2,0,4,7,14,18$, 21 and 28 of the study - Fig. 1 (He et al., 2016; Pourmohammadi et al., 2012; Sharma et al., 2006). The cut-off time was considered as 30 seconds.

\section{Tail-flick test}

The rats were restricted by a restrainer and their tails were positioned in the tail-flick apparatus (Ugo Basile, Italy). The beam of light was focused on the dorsal surface of the animal's tail. Tail-flick latency was considered as the time interval between the initiation of radiant heat stimulation and the abrupt removal of the tail from the nociceptive stimulus (cut-off time $=10 \mathrm{~s}$ ). Subsequently, the tail-flick test was performed on the same days in a manner mentioned earlier for the hot plate method. Tail-flick test was done an hour before the hot plate test (Fig. 1).

\section{Measurement of motor nerve conduction velocity}

After performing the last behavioral assessments (Fig. 1), the rats were anesthetized with thiopental (65 mg/kg, ip; Sigma, St. Louis, MO, USA). Body temperature was monitored and then maintained within normal limits. Furthermore, the motor nerve conduct velocity (MNCV) in the left sciatic nerve was measured using power lab MLT 1030/D device (AD Instruments, Power Lab, Spain) and the same stimulatory and recording pin electrodes (AD Instrument) in terms of the previously described method (Ja'afer et al., 2006). Using a bipolar hook-shaped electrode, in which its cathode was located $2 \mathrm{~mm}$ away from the anode, electrical stimulations equal to $10 \mathrm{~V}$ were introduced to the proximal section of the sciatic nerve. Compound-muscle action potentials (CMAP) were also recorded by inserting the recording and reference needle electrodes into the gastrocnemius muscle belly and calcaneus tendon, respectively. Finally, the earth electrode was located under the skin. Notably, the velocity was calculated as the ratio between the distances of stimulating and recording pin electrodes (meters) and the corresponding latency difference (seconds).

\section{Statistical analyses}

All data were expressed as Mean \pm SD (standard deviation). The obtained data were analyzed using one-way analysis of variance (ANOVA) followed by Tukey post-hoc test, and twoway ANOVA followed by Bonferroni post-hoc test. $P<0.05$ was considered as statistically significant for all analyses.

\section{Results}

\section{Blood glucose changes assessment}

Forty-eight hours after the STZ injection, blood glucose levels were assessed to confirm the induction of diabetes. Moreover, blood glucose levels measured once again on day 21 before all other tests. As shown in Fig. 2, all the study groups have a significant $(p<0.0001)$ difference in blood glucose compared to the control group (CNT). Based on our results, all the rats were diabetic except the control group.

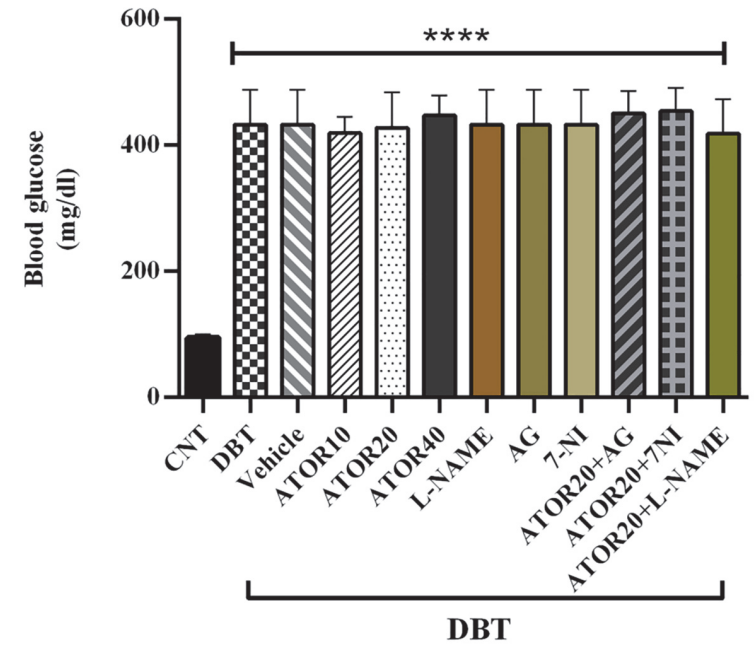

Fig. 2. Blood glucose levels. $N=6$ per studied group and data are represented as mean \pm SD. One-way ANOVA, Tukey's post-hoc test was applied for the analysis of differences in blood glucose.

**** $P<0.0001$ compared with CNT (control group). DBT (diabetic group) and ATOR (Atorvastatin).

\section{Survival study and general toxicity}

Prior to the initiation of the test, body weights were measured and no significant inter-group differences were observed. During the experiment, our findings revealed a normal weight gain pattern in the control group (Fig. 3A).

However, as shown in Fig. 3A, a significant $(p<0.0001)$ weight loss was observed in the diabetic groups (vehicle and $\mathrm{DBT}$ ) in the same early days. All the rats receiving AG, 7-NI, and L-NAME were similar to the Vehicle and DBT groups. Contrarily, it was found that co-treatment with atorvastatin at doses of 20 and $40 \mathrm{mg} / \mathrm{kg}$ prevented the effect of diabetes on body weight and significantly reduced weight loss $(p<0.05)$ (Fig 3B).

In this study, the mortality of diabetic animals was observed, but not reported due to insignificance.

\section{Thermal pain threshold assessment}

As it was mentioned earlier in the procedure, based on the classical constant-temperature, hot-plate and tail-flick tests were performed on the same days. Our results reveal that in hot plate tests, diabetic rats showed a significant decrease in paw withdrawal latency on days 7, 14, $28(p<0.05)$, $18(p<0.01)$, and $21(p<0.001)$ (Fig. 4A). Also, the tail-flick test results demonstrate that pain threshold in diabetic rats has significantly decreased on days $7,14(p<0.05), 18,21$, and 28 ( $p<0.01)$. Based on our findings, the pattern of painful diabetic neuropathy started from day 7 after the first injection of STZ, which is in agreement with previous studies (Anjaneyulu and Chopra, 2004; Balaha et al., 2018; He et al., 2016). Therefore, according to the above-mentioned results, day 21 was selected as the most productive day in hyperalgesia of painful diabetic neuropathy, and considering this, the duration of the present study was considered as 21 days. 
A

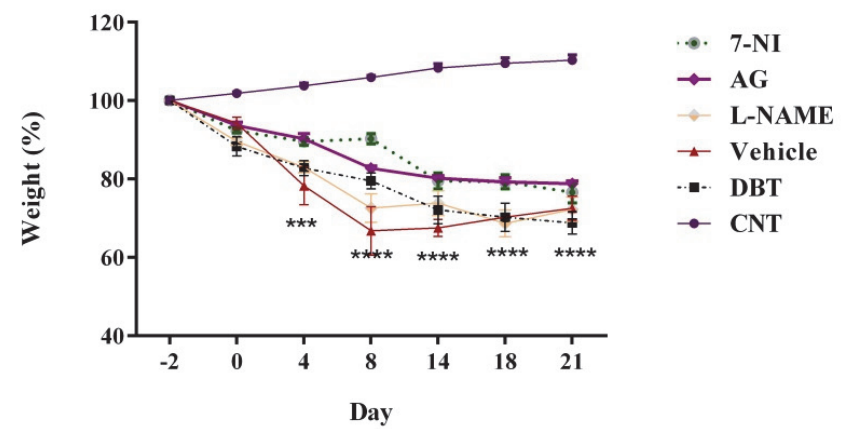

B

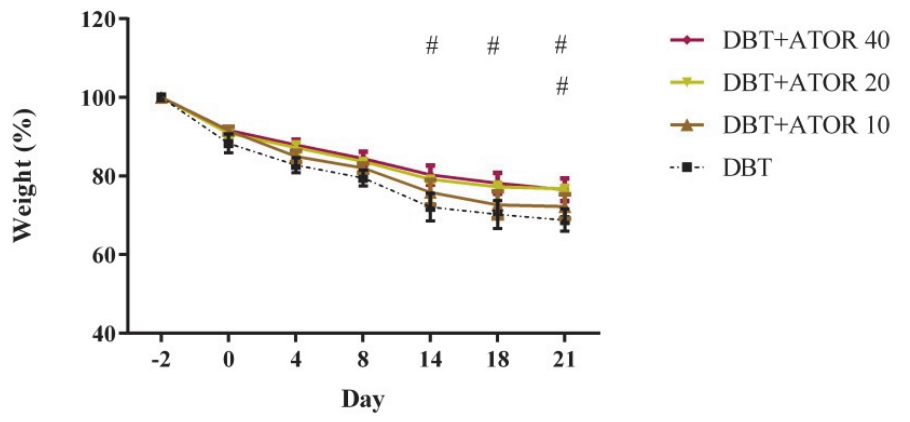

Fig. 3. Effect of different doses of atorvastatin on diabetic-induced neuropathy on weight loss. (A) Changes in rat's body weight during the 21-day period of study. Normal weight gain pattern was not observed in diabetic-induced groups and weight loss become statistically meaningful from Day 0 after the first injection of STZ. (B) Weight loss pattern was diminished in rats receiving atorvastatin and a gradual weight gain began from Day 14 following STZ administration. $N=6$ per studied group and data are represented as mean \pm SD. CNT (control group) and DBT (diabetic group). Two-way ANOVA, Bonferroni post-hoc test was applied for analysis of differences in body weights. ${ }^{* * *} P<0.001$ and ${ }^{* * * *} P<0.0001$ in comparison with the CNT group and $\# P<0.05$ compared with the DBT group. CNT (control group), DBT (diabetic group), and ATOR (Atorvastatin).

A

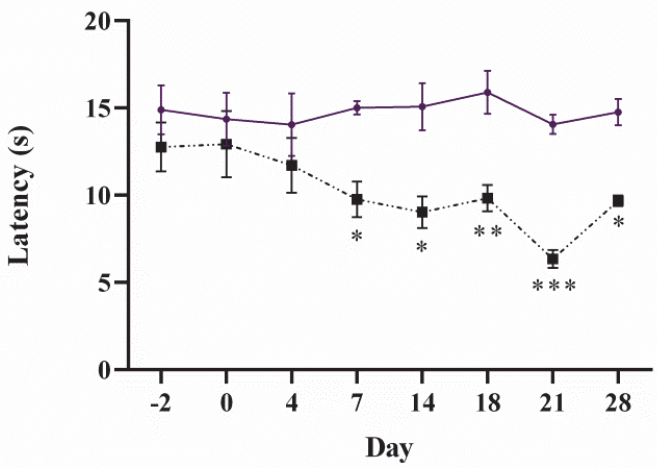

B

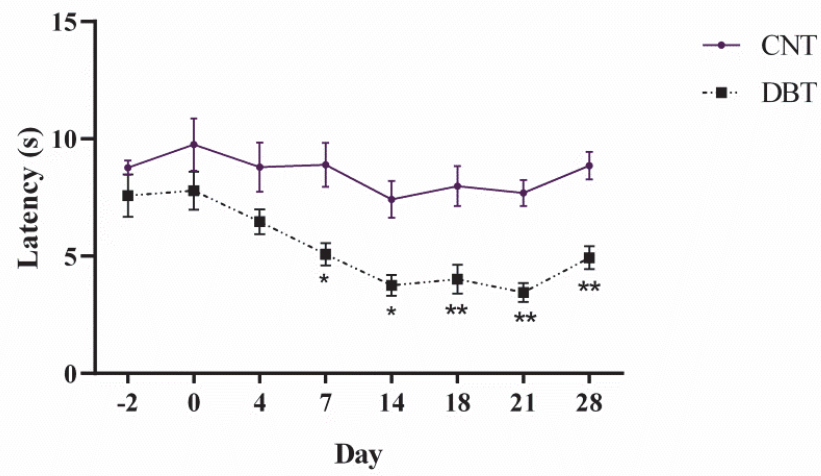

Fig 4. Time course assessment (A) Hot plate (B) Tail flick. $N=6$ per studied group and data are represented as mean \pm SD. Two-way ANOVA, Bonferroni post-hoc test was applied for the analysis of differences in thermal withdrawal latency in different days. ${ }^{* * *} P<0.001$, ${ }^{* *} P<0.01$ and ${ }^{*} P<0.05$ compared with control group (CNT). DBT (diabetic group).

\section{The different doses of atorvastatin on painful diabetic neuropathy at day 21}

The effects of different doses of atorvastatin treatment (10, 20 , and $40 \mathrm{mg} / \mathrm{kg}$ ) on thermal (heat) withdrawal thresholds are depicted in Fig. 5. Fig. 5A shows the effects of atorvastatin administration on the hot plate tests. Atorvastatin at the dose of $40 \mathrm{mg} / \mathrm{kg}$ has significantly $(p<0.001)$ reversed the pain threshold reduction caused by STZ-induced diabetes. As shown in Fig. 5A, there is a significant difference between atorvastatin at the dose of $40 \mathrm{mg} / \mathrm{kg}$, and at doses of $10(p<0.01)$ and $20 \mathrm{mg} / \mathrm{kg}(p<0.05)$. Based on this finding atorvastatin at the dose of $20 \mathrm{mg} / \mathrm{kg}$ was considered as the subeffective dose. However, further analyses revealed that there is no significant difference between the atorvastatin at the doses of 10 and $20 \mathrm{mg} / \mathrm{kg}$ and the DBT group.

Besides, the evaluation of the thermal withdrawal threshold with tail-flick tests demonstrated that the atorvastatin at the dose of $40 \mathrm{mg} / \mathrm{kg}(p<0.001)$ significantly increased the pain threshold time in comparison with the DBT (Fig. 4B). Therefore, based on these results, the effective dose of atorvastatin was considered as $40 \mathrm{mg} / \mathrm{kg}$.

\section{The effect of NOS inhibitors on painful diabetic neuropathy}

In order to evaluate the influence of the NO pathway on the neuroprotective effect of atorvastatin, the subeffective doses of atorvastatin $(20 \mathrm{mg} / \mathrm{kg})$ were administrated along with the NOS inhibitors, namely L-NAME (2 mg/kg), 7-NI (15 mg/kg), and AG (50 mg/kg). The concurrent administration of subeffective dose of atorvastatin $(20 \mathrm{mg} / \mathrm{kg})$ and NOS inhibitors unmasked a synergistic effect and the role of NO on the protective effect of atorvastatin.

The concurrent administration of L-NAME or AG with atorvastatin has significantly increased the thermal latency withdrawal in the hot plate test, indicating that the pain threshold has significantly elevated compared to the diabetic group (DBT) $(p<0.001)$. However, there was a significant difference among the ATOR20 group and the ATOR20 + LNAME $(p<0.001)$ and ATOR20 + AMINO groups $(p<0.01)$. As shown in Fig 6A, there was no significant difference between the diabetic rats receiving only NOS modulators and the diabetic group (DBT). 
A

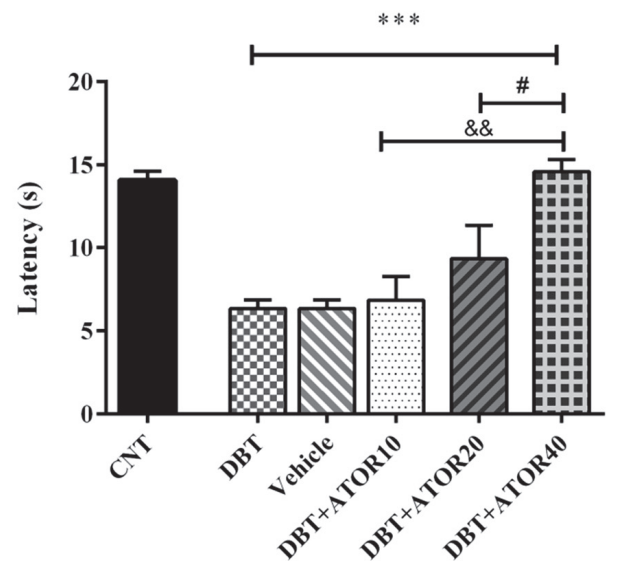

B

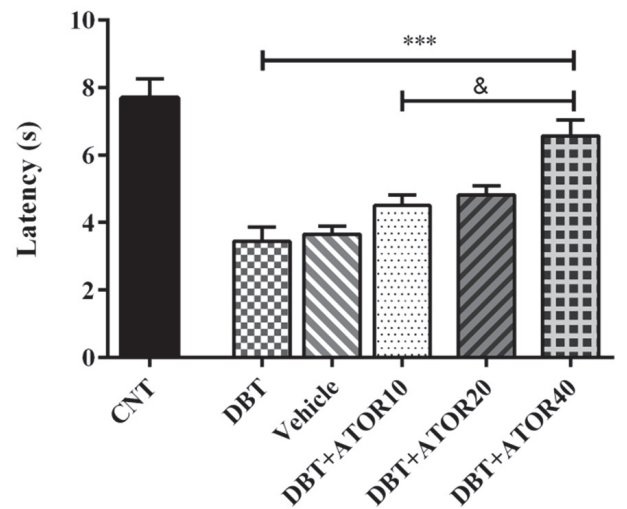

Fig. 5. The effect of different doses of atorvastatin on diabetic-induced neuropathy (A) Hot plate (B) Tail flick. $N=6$ per studied group and data are represented as mean \pm SD. One-way ANOVA, Tukey's post-hoc test was applied for the analysis of differences in thermal withdrawal latency at day $21 .{ }^{* *} P<0.001$ and ${ }^{*} P<0.05$ compared with DBT. \# $P<0.05$ compared with DBT + ATOR20. \& $P<0.05$ and $\& \& P<0.001$ compared with DBT+ ATOR10. CNT (control group), DBT (diabetic group), and ATOR (Atorvastatin).

Fig. 6B depicts the effects of the co-administration of the NOS inhibitors and atorvastatin in the tail-flick test. Our findings revealed that the concomitant administration of L-NAME, AG, or 7-NI with atorvastatin caused a significant effect on the pain threshold. In other words, the co-treatment with atorvastatin and NOS inhibitors has significantly elevated the with- drawal latency compared to the diabetic group (DBT). Also, there was a significant difference between the ATOR20 group and the ATOR20 + LNAME $(p<0.01)$. However, L-NAME (2 mg/kg), 7-NI (15 mg/kg), and AG (50 mg/kg) administrations alone did not alter the analgesic threshold in diabetic rats compared to the diabetic group (DBT).
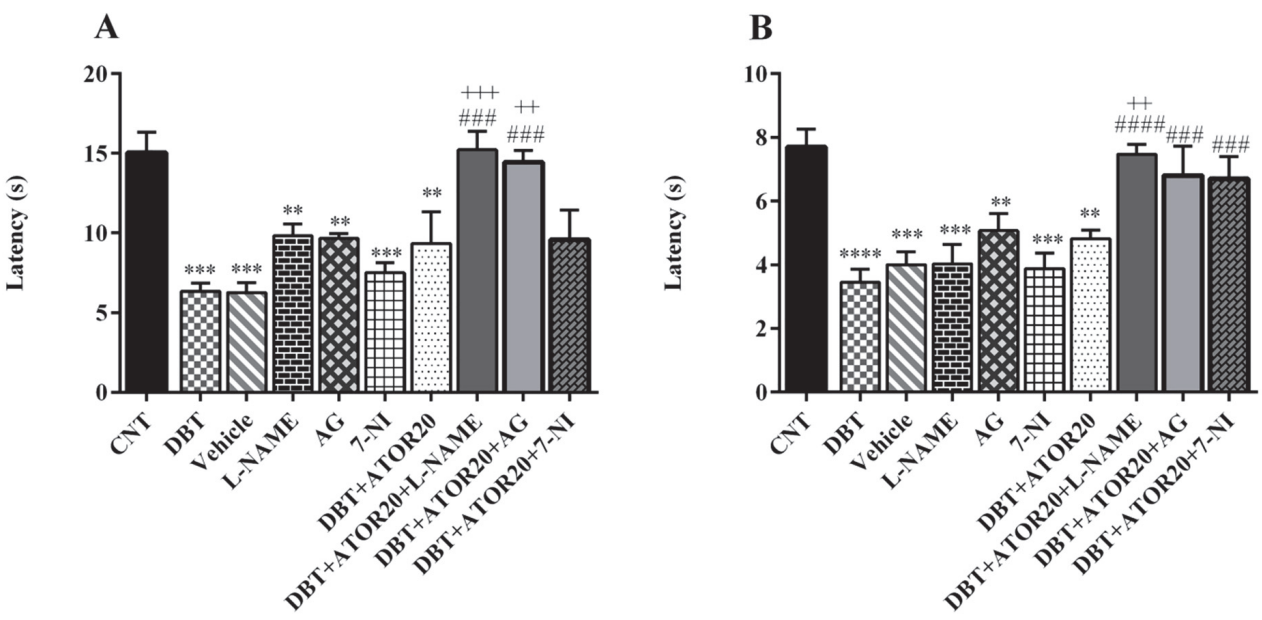

Fig. 6. The effect of NOS modulators L-NAME ( $2 \mathrm{mg} / \mathrm{kg})$, 7-NI (15 mg/kg), AG (50 mg/kg) on diabetic-induced neuropathy (A) Hot plate (B) Tail flick. $N=6$ per studied group and data are represented as mean \pm SD. One-way ANOVA, Tukey's post-hoc test was applied for the analysis of differences in thermal withdrawal latency at day $21 .{ }^{* *} P<0.01,{ }^{* *} P<0.001$ and ${ }^{* * * *} P<0.0001$ compared with CNT, \#\#\# $P<0.001$ and \#\#\#\# $P<0.0001$ compared with DBT, $++P<0.01$ and $+++P<0.001$ compared with ATOR20. CNT (control group), DBT (diabetic group), and ATOR (Atorvastatin).

\section{The effect of atorvastatin on MNCV in painful diabetic neuropathy}

As shown in Fig.7A, MNCV has significantly reduced in the DBT rats in comparison with the CNT ones $(P<0.001)$. Additionally, the administration of atorvastatin at the dose of $40 \mathrm{mg} / \mathrm{kg}$ altered a significant $(P<0.05)$ reversal of the motor nerve conduction velocity compared with the DBT, while atorvastatin at the doses of 10 and $20 \mathrm{mg} / \mathrm{kg}$ showed no significant effect.
Moreover, the co-administration of L-NAME and subeffective dose of atorvastatin $(20 \mathrm{mg} / \mathrm{kg})$ has significantly $(P<0.01)$ increased the MNCV in comparison with the DBT group. Furthermore, the co-administration of L-NAME and atorvastatin led to a significant $(P<0.05)$ potentiation of MNCV compared to the DBT +ATOR 20 group. In contrast, $\mathrm{DBT}+\mathrm{ATOR} 20+7 \mathrm{NI}$ and DBT + ATOR20 + AMINO groups, sciatic MNCV, had no significant differences with the atorvastatin group $(P>0.05)$ (Fig. 7B). 
A

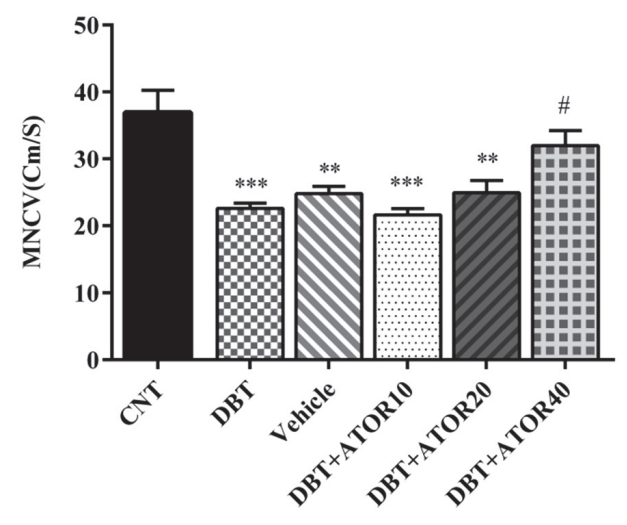

B

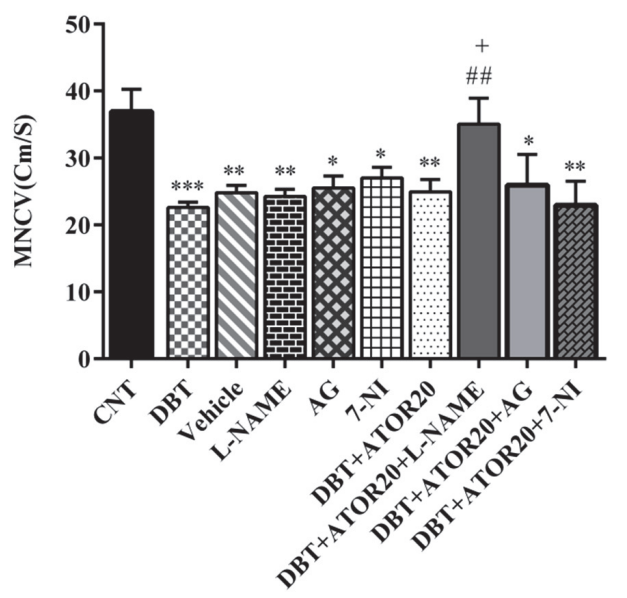

Fig 7. (A) The effects of different doses of atorvastatin and (B) its co-administration with NOS modulators L-NAME (2 mg/kg), 7-NI $(15 \mathrm{mg} / \mathrm{kg}), \mathrm{AG}(50 \mathrm{mg} / \mathrm{kg})$ on MNCV in diabetic rats. $N=6$ per studied group and data are represented as mean \pm SD. One-way ANOVA followed by Tukey's post-hoc test. ${ }^{*} P<0.05,{ }^{* *} P<0.01$ and ${ }^{* * *} P<0.001$ compared with CNT, \# $P<0.05$ and \#\# $P<0.01$ compared with DBT, $+P<0.05$ compared with DBT+ATOR20.

\section{Discussion}

Peripheral neuropathy is one of the most common complications caused by diabetes, which is manifested in the form of spontaneous pain, allodynia, and hyperalgesia as a result of an injury to the somatosensory system (Pathak et al., 2013; ten Donkelaar et al., 2020; Themistocleous et al., 2016). This painful signal is believed to originate in the peripheral nervous system; however, the exact mechanisms underlying diabetic allodynia and hyperalgesia still remain poorly understood ( $\mathrm{Li}$ et al., 2015). Correspondingly, the treatment of neuropathic pain becomes a challenging task for clinicians due to its complex pathophysiology and severe adverse effects of the current conventional agents (Pathak et al., 2013).

This study aimed to investigate the protective effects of atorvastatin on painful diabetes' neuropathy. Based on the findings of this study, it can be suggested that atorvastatin could reduce NOS activity due to diabetes mellitus. In the present study, it has been shown that the atorvastatin group at the dose of $40 \mathrm{mg} / \mathrm{kg}$ decreased hyperalgesia and painful progression in the STZ-induced diabetic rats. Several factors contributing to the pathogenesis of diabetic neuropathy provide a wide range of goals for potential therapeutic interventions. A comprehensive research has shown that overproduction of nitric oxide by the nervous and non-nervous systems plays an essential role in the multiple pathologies of peripheral neuropathy (Huang et al., 2015). In this study, by performing behavioral and electrophysiological assessments, it was indicated that atorvastatin ameliorates neuropathic pain and attenuates the diabetes-induced hyperalgesia. Behavioral studies, including hot plate and tail-flick tests, were also performed to evaluate hyperalgesia. It is noteworthy that in diabetes, hyperglycemia can cause the abnormal metabolism of the peripheral nervous system, which consequently leads to deficiencies in the nervous function and the reduced MNCV. Furthermore, nerve fibers exhibit severe demyelination, with most of the large axons being surrounded either by very few turns of myelin or by none at all (Yao et al., 2018). According to our findings, a significant decrease in MNCV was observed in the sciatic nerve in the diabetic group. Relying on the results of thermal hyperalgesia and MNCV tests, our findings revealed that atorvastatin had improved the function of small and large nerve fibers. Based on the previous reports on the efficacy of statin therapy, the atorvastatin administration in this study showed fewer symptoms of neuropathy and also prevented sensory-motor neuropathy in behavioral and electrophysiological studies. Our results demonstrate that the atorvastatin treatment in higher doses could bring significant antinociceptive effects on diabetic rats. Additionally, this study confirmed the involvement of the nitric oxide system in diabetic's neuropathy. These findings are consistent with those of the previous studies reporting the involvement of NO (Boels et al., 2017; Minaz and Razdan, 2016), an important neuromodulator that is thought to play a dual role as both nociceptive and anti-nociceptive under neuropathic pain conditions (Cury et al., 2011; Huang et al., 2015). Different studies have pointed out the prominent role of neuronal nitric oxide synthase (nNOS) in the development and pathology of both peripheral and central neuropathic pains. Injuries in peripheral nerves result in overexpression of nNOS in rat's spinal cord, which is associated with hyperalgesia and allodynia (Čížková et al., 2002; Jin et al., 2009; Rondón et al., 2018). It has been shown that systemic or intrathecal administration of the selective or nonselective nNOS inhibitors is associated with the reduced sensation of pain in neuropathic pain (Cury et al., 2011; Rocha et al., 2020).

Concerning the effect of NO on nociceptive transmission, there are conflicting data. Considerable evidences show that NO can cause pain, intensify inflammatory hyperalgesia, and promote neuropathic pain (Staunton et al., 2018), but it can also produce anti-nociception (Gomes et al., 2020; Kusuda et al., 2020). The dual effects of NO in inflammatory pain may be due to different parameters such as pain models, testing paradigms, and doses of NO donors and inhibitors. (Chen et al., 2010; Cury et al., 2011; Kusuda et al., 2020; Stavniichuk et al., 2014). In the present study, L-NAME pre-treatment with subeffective dose of atorvastatin caused potentiation of the anti-hyperalgesic effect of atorvastatin in both hot plate and tail-flick tests, suggesting the possible involvement of NO 
pathway in its protective action. In addition, chronic administration of LNAME with atorvastatin caused a significant increase in MNCV compared to the atorvastatin-treated group. To investigate the possible involvement of NOS isoforms, we examined the chronic co-administration of iNOS and nNOS inhibitors with subeffective dose of atorvastatin. These results indicate that both AG and 7NI could enhance the analgesic threshold of atorvastatin in diabetic rats. In general, it can be stated that the inhibition of the NOS pathway reduces nitric oxide. Additionally, co-administration of atorvastatin with NOS inhibitors synergistically acts and consequently improves neuropathy. Several papers provided evidence on a complex linkage between atorvastatin and NO production (Aydin et al., 2013; Gomes et al., 2020). However, there are conflicting reports around the role of NO in the mechanism of action of statins. While it has been shown that atorvastatin significantly elevates the NO production in endothelial cells (El-Sisi et al., 2013), other reports have shown that atorvastatin has anti-inflammatory effects through NO inhibition (Chu et al., 2015; Kandasamy et al., 2011; McGown et al., 2015; Wagner et al., 2002). Previous reports have shown that statins operates as an effective agent against various complications through the involvement of nitric oxide (Ferro et al., 2000; Giusti-Paiva et al., 2004; Hernández-Romero et al., 2008; Pahan et al., 1997). Of note, statins were recognized as being capable of inhibiting the activation of inflammatory factors including inducible nitric oxide synthase (iNOS), as well as oxidative stress, which in turn can attenuate the neuropathic pain (Aydin et al., 2013; Pathak et al., 2014).

Despite the cholesterol-reducing actions of HMG-CoA reductase inhibitors (statins), they have recently been shown to exert beneficial effects on attenuating neuropathic pain in different disease states and various animal models of neuropathy (Bhalla et al., 2015; Hassanipour et al., 2016; Shi et al., 2011). Garcia et al. (2011) reported that the daily administration of atorvastatin resulted in some significant anti-nociceptive effects on the tail flick and formalin tests (Chu et al., 2015; Garcia et al., 2011). Metabolic disorders in diabetes appear to affect neural function through vascular effects (Duarte, 2015). Previous reports have shown that rosuvastatin could affect the neurodevelopmental disorder of diabetes by affecting the system containing large and small nerve fibers. Improvement in neural tissue perfusion appears as a vital mechanism related to these effects (Pathak et al., 2013; Shi et al., 2011). Rosuvastatin affects both nitrergic system and endothelial hyperpolarization, which also improves blood flow to the nervous system (Pathak et al., 2013).

In 2014, Pathak et al. reported that atorvastatin prescribed at all doses used in the study did not affect peritoneal and supraspinal levels of nitrite in peripheral neuropathy. However, despite increasing levels of nitrite, atorvastatin decreased hyperalgesia at the end of the study, suggesting that hyperalgesia is not merely associated with the increased NO levels in the central nervous system (Pathak et al., 2014). In the study, the anti-hyperalgesic effect of atorvastatin demonstrated the role of monoxide and peroxynitrite in persistent pain. The peroxynitrite precursor of monoxide has significantly decreased by atorvastatin and this observation was associated with the direct effect of atorvastatin on monoxide as well as on the reduction of oxidative stress (Pathak et al., 2014). Based on the previous studies, peroxynitrite is biosynthesized by the reaction of NO with diabetes-induced superoxide. Peroxynitrite is a neurotoxic molecule involved in neuropathic pain pathology (Pacher et al., 2007; Stavniichuk et al., 2014). In accordance with the previous studies, our results demonstrate that chron- ic treatment with atorvastatin showed a significant anti-nociceptive effect on diabetic rats. Atorvastatin administration for a 21-day period improved the small and large nerve fiber defects (that were assessed using thermal hyperalgesia tests and sciatic MNCV test).

\section{Conclusions}

In conclusion, our study provided insight into the notion that atorvastatin attenuates diabetic hyperalgesia and the fact that nitric oxide mechanism could be involved in the neuroprotective effect of atorvastatin. Furthermore, the study highlighted the therapeutic potential of atorvastatin in the management of diabetic neuropathic pain and the related conditions. Further molecular and cellular investigations are needed to clarify the exact mechanism of action of statins in order to enlighten pathways in the treatment of neuropathic pain.

\section{Conflict of interests}

The authors have no conflict of interests to declare.

\section{Acknowledgements}

This study was part of $\mathrm{PhD}$ thesis and was funded and supported by a grant from Department of Pharmacology, School of Medicine, Tehran University of Medical Sciences (TUMS), grant no. 94-03-30-29187; and Iran National Science Foundation (INSF); grant no. 96002757.

\section{References}

Ahlawat A, Sharma S (2018). A new promising simultaneous approach for attenuating type II diabetes mellitus induced neuropathic pain in rats: iNOS inhibition and neuroregeneration. Eur J Pharmacol 818: 419-428. DOI: 10.1016/j. ejphar.2017.11.010

Anjaneyulu M, Chopra K (2004). Quercetin attenuates thermal hyperalgesia and cold allodynia in STZ-induced diabetic rats. Indian J Exp Biol 42: 766-769.

Aydin Ş, Yildirim E, Erol K (2013). Effects of simvastatin on experimental neuropathic pain model and the role of nitric oxide. J Analgesics 1: 1-8.

Balaha M, Kandeel S, Kabel A (2018). Phloretin either alone or in combination with duloxetine alleviates the STZ-induced diabetic neuropathy in rats. Biomed Pharmacother 101: 821-832. DOI: 10.1016/j.biopha.2018.02.135.

Bhalla S, Singh N, Jaggi AS (2015). Dose-related neuropathic and anti-neuropathic effects of simvastatin in vincristineinduced neuropathic pain in rats. Food Chem Toxicol 80: 32-40. DOI: 10.1016/j.fct.2015.02.016.

Boels MGS, van Faassen EEH, Avramut MC, van der Vlag J, van den Berg BM, Rabelink TJ (2017). Direct observation of enhanced nitric oxide in a murine model of diabetic nephropathy. PloS One 12(1): e0170065. DOI: 10.1371/journal.pone.0170065.

Chen Y, Boettger MK, Reif A, Schmitt A, Üçeyler N, Sommer C (2010). Nitric oxide synthase modulates CFA-induced thermal hyperalgesia through cytokine regulation in mice. Mol Pain 6: 13 DOI: 10.1186/1744-8069-6-13.

Chu L-W, Chen J-Y, Wu P-C, Wu B-N (2015). Atorvastatin prevents neuroinflammation in chronic constriction injury rats through nuclear NFkB downregulation in the dorsal root ganglion and spinal cord. ACS Chem Neurosci 6(6): 889-898. DOI: 10.1021/ acschemneuro.5b00032.

Čížková D, Lukáčová N, Maršala M, Maršala J (2002). Neuropathic pain is associated with alterations of nitric oxide synthase immunoreactivity and catalytic activity in dorsal root ganglia and 
spinal dorsal horn. Brain Res Bull 58(2): 161-171. DOI: 10.1016/ s0361-9230(02)00761-x.

Cury Y, Picolo G, Gutierrez VP, Ferreira SH (2011). Pain and analgesia: the dual effect of nitric oxide in the nociceptive system. Nitric Oxide 25(3): 243-254. DOI: 10.1016/j.niox.2011.06.004.

de Los Monteros-Zuñiga AE, Izquierdo T, Quiñonez-Bastidas GN, Rocha-González HI, Godínez-Chaparro B (2016). Anti-allodynic effect of mangiferin in neuropathic rats: Involvement of nitric oxide-cyclic GMP-ATP sensitive $\mathrm{K}+$ channels pathway and serotoninergic system. Pharmacol Biochem Behav 150: 190-197. DOI: 10.1016/j.pbb.2016.10.007.

Duarte JMN (2015). Metabolic alterations associated to brain dysfunction in diabetes. Aging Dis 6(5): 304-321. DOI: 10.14336/ AD.2014.1104.

Eitah HE, Maklad YA, Abdelkader NF, El Din AAG, Badawi MA, Kenawy SA (2019). Modulating impacts of quercetin/sitagliptin combination on streptozotocin-induced diabetes mellitus in rats. Toxicol Appl Pharmacol 365: 30-40. DOI: 10.1016/j. taap.2018.12.011.

El-Sisi A, Hegazy SK, Salem KA, AbdElkawy KS (2013). Atorvastatin improves erectile dysfunction in patients initially irresponsive to Sildenafil by the activation of endothelial nitric oxide synthase. Int J Impot Res 25(4): 143-148. DOI: 10.1038/ijir.2012.46.

Ferro D, Parrotto S, Basili S, Alessandri C, Violi F (2000). Simvastatin inhibits the monocyte expression of proinflammatory cytokines in patients with hypercholesterolemia. J Am Coll Cardiol 36(2): 427-431. DOI: 10.1016/s0735-1097(00)00771-3.

Garcia GG, Miranda HF, Noriega V, Sierralta F, Olavarría L, Zepeda RJ, Prieto JC (2011). Antinociception induced by atorvastatin in different pain models. Pharmacol Biochem Behav 100(1): 125-129. DOI: 10.1016/j.pbb.2011.08.007.

Giusti-Paiva A, Martinez MR, Felix JVC, da Rocha MJA, Carnio EC, Elias LLK, Antunes-Rodrigues J (2004). Simvastatin decreases nitric oxide overproduction and reverts the impaired vascular responsiveness induced by endotoxic shock in rats. Shock 21(3): 271-275. DOI: 10.1097/10.shk.0000115756.74059.ce.

Gomes FIF, Cunha FQ, Cunha TM (2020). Peripheral nitric oxide signaling directly blocks inflammatory pain. Biochem Pharmacol 176: 113862. DOI: 10.1016/j.bcp.2020.113862.

Hai-Na Z, Xu-Ben Y, Cong-Rong T, Yan-Cheng C, Fan Y, Lei-Mei X, et al. (2020). Atorvastatin ameliorates depressive behaviors and neuroinflammatory in streptozotocin-induced diabetic mice. Psychopharmacology (Berl) 237(3): 695-705. DOI: 10.1007/ s00213-019-05406-w.

Hasanvand A, Amini-Khoei H, Hadian M-R, Abdollahi A, Tavangar SM, Dehpour AR, et al. (2016). Anti-inflammatory effect of AMPK signaling pathway in rat model of diabetic neuropathy. Inflammopharmacology 24(5): 207-219. DOI: 10.1007/s10787016-0275-2.

Hassanipour M, Amini-Khoei H, Shafaroodi H, Shirzadian A, Rahimi N, Imran-Khan M, et al. (2016). Atorvastatin attenuates the antinociceptive tolerance of morphine via nitric oxide dependent pathway in male mice. Brain research bulletin 125: 173-180. DOI: 10.1016/j.brainresbull.2016.07.002.

He W-Y, Zhang B, Xiong Q-M, Yang CX, Zhao W-C, He J, et al. (2016). Intrathecal administration of rapamycin inhibits the phosphorylation of DRG Nav1. 8 and attenuates STZ-induced painful diabetic neuropathy in rats. Neurosci Lett 619: 21-28. DOI: 10.1016/j.neulet.2016.02.064

He W-Y, Zhang B, Zhao W-C, He J, Zhang L, Xiong Q-M, et al. (2019). Contributions of mTOR Activation-Mediated Upregulation of Synapsin II and Neurite Outgrowth to Hyperalgesia in STZInduced Diabetic Rats. ACS Chem Neurosci 10(5): 2385-2396. DOI: 10.1021 /acschemneuro.8b00680.

Heltianu C, Guja C (2011). Role of nitric oxide synthase family in diabetic neuropathy. J Diabetes Metab 5: 002. DOI: 10.4172/2155-6156.S5-002.

Hernández-Romero M del C, Argüelles S, Villarán RF, de Pablos RM, Delgado-Cortés MJ, Santiago M, et al. (2008). Simvastatin prevents the inflammatory process and the dopaminergic degeneration induced by the intranigral injection of lipopolysaccharide. J Neurochem 105(2): 445-459. DOI: 10.1111/j.1471-4159.2007.05148.x.
Huang LY, Tsui DY, Williams CM, Wyse BD, Smith MT (2015). The furoxan nitric oxide donor, PRG150, evokes dose-dependent analgesia in a rat model of painful diabetic neuropathy. Clin Exp Pharmacol Physiol 42(9): 921-929. DOI: 10.1111/14401681.12442

Ja'afer FMH, Hamdan FB, Mohammed FH (2006). Vincristineinduced neuropathy in rat: electrophysiological and histological study. Exp Brain Res 173(2): 334-345. DOI: 10.1007/s00221-0060499-2.

Jesus CHA, Redivo DDB, Gasparin AT, Sotomaior BB, de Carvalho MC, Genaro K, et al. (2019). Cannabidiol attenuates mechanical allodynia in streptozotocin-induced diabetic rats via serotonergic system activation through 5-HT1A receptors. Brain Res 1715: 156-164. DOI: 10.1016/j.brainres.2019.03.014.

Jin X-G, He S-Q, Yan X-T, Zhang G, Wan L, Wang J, et al. (2009). Variants of Neural Nitric Oxide Synthase in the Spinal Cord of Neuropathic Rats and Their Effects on Nuclear Factor- $\kappa B$ (NFкB) Activity in PC12 Cells. J Pain 10(1): 80-89. DOI: 10.1016/j. jpain.2008.07.009.

Kandasamy K, Prawez S, Choudhury S, More AS, Ahanger AA, Singh TU, et al. (2011). Atorvastatin prevents vascular hyporeactivity to norepinephrine in sepsis: role of nitric oxide and a1-adrenoceptor mRNA expression. Shock 36(1): 76-82. DOI: 10.1097/SHK.0b013e31821a4002.

Kaur S, Pandhi P, Dutta P (2011). Painful diabetic neuropathy: an update. Ann Neurosci 18(4): 168-175. DOI: 10.5214/ans.09727531.1118409.

Kusuda R, Carreira EU, Ulloa L, Cunha FQ, Kanashiro A, Cunha TM (2020). Choline attenuates inflammatory hyperalgesia activating nitric oxide/cGMP/ATP-sensitive potassium channels pathway. Brain Res 1727: 146567. DOI: 10.1016/j.brainres.2019.146567.

Li M-Y, Wang Y-Y, Cao R, Hou X-H, Zhang L, Yang R-H, Wang F (2015). Dietary fish oil inhibits mechanical allodynia and thermal hyperalgesia in diabetic rats by blocking nuclear factor$\kappa B$-mediated inflammatory pathways. J Nutr Biochem 26(11): 1147-1155. DOI: 10.1016/j.jnutbio.2015.05.005.

Mbiantcha M, Khalid R, Atsamo DA, Njoku IS, Mehreen A, Ateufack G, et al. (2020). Anti-hypernociceptive effects of methanol extract of Boswellia dalzielii on STZ-induced diabetic neuropathic pain. Adv Tradit Med 1-13. DOI: 10.1007/s13596019-00411-y.

McGown CC, Brookes ZLS, Hellewell PG, Ross JJ, Brown NJ (2015). Atorvastatin reduces endotoxin-induced microvascular inflammation via NOSII. Naunyn Schmiedeberg's Arch Pharmacol 388(5): 557-564. DOI: 10.1007/s00210-015-1100-y.

Minaz N, Razdan R (2016). Therapeutic insight into molsidomine, a nitric oxide donor in streptozotocin-induced diabetic nephropathy in rats. Indian J Pharmacol 48(5): 544-549. DOI: 10.4103/0253-7613.190744.

Newman CB, Szarek M, Colhoun HM, Betteridge DJ, Durrington PN, Hitman GA, et al. (2008). The safety and tolerability of atorvastatin $10 \mathrm{mg}$ in the Collaborative Atorvastatin Diabetes Study (CARDS). Diab Vasc Dis Res 5(3): 177-183. DOI: 10.3132/ dvdr.2008.029.

Pacher P, Beckman JS, Liaudet L (2007). Nitric oxide and peroxynitrite in health and disease. Physiol Rev 87(1): 315-424. DOI: 10.1152/physrev.00029.2006.

Pahan K, Sheikh FG, Namboodiri A, Singh I (1997). Lovastatin and phenylacetate inhibit the induction of nitric oxide synthase and cytokines in rat primary astrocytes, microglia, and macrophages. J Clin Invest 100(11): 2671-2679. DOI: 10.1172/JCI119812.

Pathak NN, Balaganur V, Lingaraju MC, Kant V, Latief N, More AS, et al. (2014). Atorvastatin attenuates neuropathic pain in rat neuropathy model by down-regulating oxidative damage at peripheral, spinal and supraspinal levels. Neurochem Int 68: 1-9. DOI: 10.1016/j.neuint.2014.01.014.

Pathak NN, Balaganur V, Lingaraju MC, More AS, Kant V, Kumar D, et al. (2013). Antihyperalgesic and anti-inflammatory effects of atorvastatin in chronic constriction injury-induced neuropathic pain in rats. Inflammation 36(6): 1468-1478. DOI: 10.1007/ s10753-013-9688-x.

Pourmohammadi N, Alimoradi H, Mehr SE, Hassanzadeh G, Hadian MR, Sharifzadeh M, et al. (2012). Lithium attenuates 
peripheral neuropathy induced by paclitaxel in rats. Basic Clin Pharmacol Toxicol 110(3): 231-237. DOI: 10.1111/j.17427843.2011.00795.x.

Raikou M, McGuire A, Colhoun HM, Betteridge DJ, Durrington PN, Hitman GA, et al. (2007). Cost-effectiveness of primary prevention of cardiovascular disease with atorvastatin in type 2 diabetes: results from the Collaborative Atorvastatin Diabetes Study (CARDS). Diabetologia 50(4): 733-740. DOI: 10.1007/ s00125-006-0561-4.

Rashidian A, Muhammadnejad A, Dehpour A-R, Mehr SE, Akhavan MM, Shirkoohi R, et al. (2016). Atorvastatin attenuates TNBS-induced rat colitis: the involvement of the TLR4/NF-kB signaling pathway. Inflammopharmacology 24(2-3): 109-118. DOI: 10.1007/s10787-016-0263-6.

Rocha PA, Ferreira AFB, Da Silva JT, Alves AS, Martins DO, Britto LRG, Chacur M (2020). Effects of selective inhibition of nNOS and iNOS on neuropathic pain in rats. Mol Cell Neurosci 105: 103497. DOI: 10.1016/j.mcn.2020.103497.

Rondón LJ, Farges MC, Davin N, Sion B, Privat AM, Vasson MP, et al. (2018). L-Arginine supplementation prevents allodynia and hyperalgesia in painful diabetic neuropathic rats by normalizing plasma nitric oxide concentration and increasing plasma agmatine concentration. Eur J Nutr 57(7): 2353-2363. DOI: 10.1007/ s00394-017-1508-x.

Rosenberger DC, Blechschmidt V, Timmerman H, Wolff A, Treede R-D (2020). Challenges of neuropathic pain: focus on diabetic neuropathy. J Neural Transm (Vienna) 127(4): 589-624. DOI: 10.1007/s00702-020-02145-7.

Rostamian A, Gharedaghi A, Norouzi-Javidan A, Dehpour AR (2019). Involvement of the nitric oxide pathway in the anti-depressantlike effects of thalidomide in mice. Physiol Behav 208: 112572. DOI: 10.1016/j.physbeh.2019.112572.

Saffarpour S, Nasirinezhad F (2019). Ascorbic acid eliminated pain-induced peripheral neuropathy by modulation of nitric oxide pathway in rats. Nutrire 44(2): 8. DOI: 10.1186/s41110-0190098-1.

Saha RN, Pahan K (2006). Regulation of inducible nitric oxide synthase gene in glial cells. Antioxid Redox Signal 8(5-6): 929-947. DOI: 10.1089/ars.2006.8.929.

Shafaroodi H, Moezi L, Fakhrzad A, Hassanipour M, Rezayat M, Dehpour AR (2012). The involvement of nitric oxide in the anti- seizure effect of acute atorvastatin treatment in mice. Neurol Res 34(9): 847-853. DOI: 10.1179/1743132812Y.0000000080.

Sharma, S, Kulkarni SK, Agrewala JN, Chopra K (2006). Curcumin attenuates thermal hyperalgesia in a diabetic mouse model of neuropathic pain. Eur J Pharmacol 536(3): 256-261. DOI: 10.1016/j.ejphar.2006.03.006.

Shi XQ, Lim TKY, Lee S, Zhao YQ, Zhang J (2011). Statins alleviate experimental nerve injury-induced neuropathic pain. Pain 152(5): 1033-1043. DOI: 10.1016/j.pain.2011.01.006.

Staunton CA, Barrett-Jolley R, Djouhri L, Thippeswamy T (2018). Inducible nitric oxide synthase inhibition by $1400 \mathrm{~W}$ limits pain hypersensitivity in a neuropathic pain rat model. Exp Physiol 103(4): 535-544. DOI: 10.1113/EP086764.

Stavniichuk R, Shevalye H, Lupachyk S, Obrosov A, Groves JT, Obrosova IG, Yorek MA (2014). Peroxynitrite and protein nitration in the pathogenesis of diabetic peripheral neuropathy. Diabetes Metab Res Rev 30(8): 669-678. DOI: 10.1002/ dmrr.2549.

Sun W, Miao B, Wang X-C, Duan J-H, Ye X, Han W-J, et al. (2012). Gastrodin inhibits allodynia and hyperalgesia in painful diabetic neuropathy rats by decreasing excitability of nociceptive primary sensory neurons. PLoS One 7(6): e39647. DOI: 10.1371/journal. pone.0039647.

ten Donkelaar HJ, Broman J, van Domburg P (2020). The somatosensory system. Clinical Neuroanatomy, Springer: 171-255. DOI: 10.1007/978-3-030-41878-6_4.

Themistocleous AC, Ramirez JD, Shillo PR, Lees JG, Selvarajah D, Orengo C, et al. (2016). The Pain in Neuropathy Study (PiNS): a cross-sectional observational study determining the somatosensory phenotype of painful and painless diabetic neuropathy. Pain 157(5): 1132-1145. DOI: 10.1097/j. pain.0000000000000491.

Wagner AH, Schwabe O, Hecker M (2002). Atorvastatin inhibition of cytokine-inducible nitric oxide synthase expression in native endothelial cells in situ. Br J Pharmacol 136(1): 143-149. DOI: 10.1038/sj.bjp.0704678.

Yao H, Feng J, Zheng Q, Wie Y, Yang G, Feng W (2018). Comparison of the Effects of Prophylactic and Therapeutic Administrations on Peripheral Neuropathy in Streptozotocin-Diabetic Rats with Gliclazide or Methylcobalamin. Exp Clin Endocrinol Diabetes 128(10):635-643. DOI: 10.1055/a-0635-0672. 